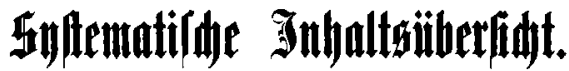

(Erite Mbtheilung. Eeite

Einleitende Betradytungen . . . . . . . . . . . . $\quad$. 1

Bucitc Mbthcilüg.

Bon ben Berfonen $. \quad . \quad . \quad . \quad . \quad . \quad . \quad 21$

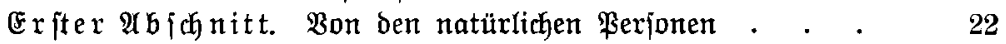

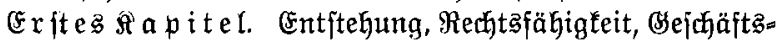

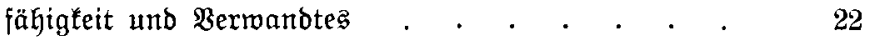

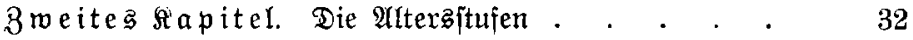

Drittes sapitel. Wolljährigleitserflärung und Ent= münbigung . . . . . . . . . . . 38

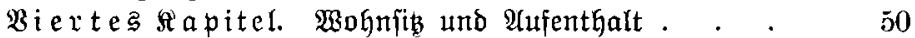

Fünfte $\mathfrak{A}$ apitel. Berjünlichfeitsechte, insbejondere Ramenbredft . . . . . . . . . . . 60

Sediftes Siatel. Tob und Tobeserklärung . . 85

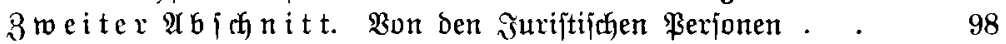

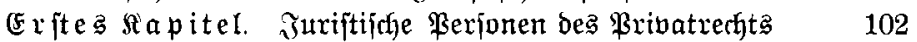

I. Bereine . . . . . . 102

II. Stiftungen . . . . . 144

8weites $\mathfrak{x}$ apitel. Surtiftiche \$exjonen bes öffent=

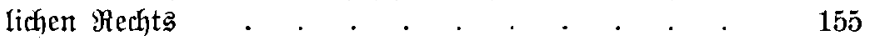

\section{Dritte Ylotbeilüg.}

Bon bex familie . . . . . . . . 174

Exiter $\mathfrak{A b}$ f nitt. Eherechtliche Berbältnifie . . . . 174

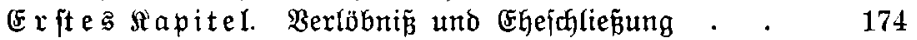

3 we i tes $\mathfrak{x} \mathfrak{a} \mathfrak{p}$ i tel. Kagemeinredthtiche Wixtungen ber Ehe . . . . . . . . . . 203

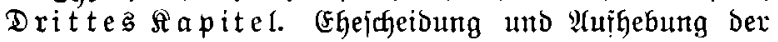
ehelichen Bremeinichajt 


\section{$\underline{\mathrm{X}}$}

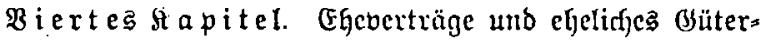
redft . . . . . . . . . . . . 216

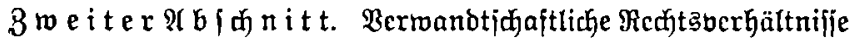

Erite 3 sapitel. Berwandtihaft unb Erfwägerjanft im Yagemeinen . . . . . . . . .

3weites stapitel. Redftaverhältniffe ber eljelidfen finber . . . . . . . . . . . 230

Drittez Siatel. Mnnahme an Sindeşintt . : $\quad 250$

Ziertes $\mathfrak{H}$ a pitel. Itnterhaltspplicht . . . . 253

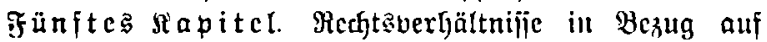
uneljeliche Stinder $\quad . \quad . \quad . \quad . \quad . \quad . \quad . \quad 267$

Dritter Mbjunitt. Bormundidaftatwejen. . . 284

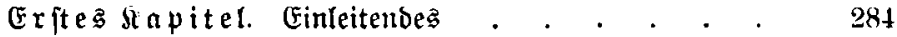

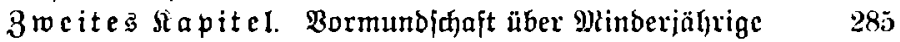

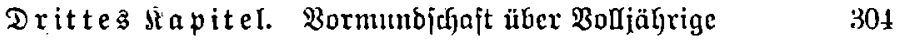

Biertes sapitcl. Pflcgichaft . . . . . . $\quad 307$

\section{Bicrte Mbthetluna.}

Won Saden und Gadcuredten. . . 310

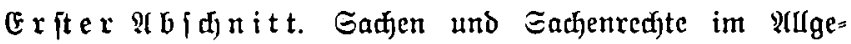
meinen . . . . . . . . . 311

Exftes fiapitel. Einleitendes . . . . . . 311

3 we it es sapitel. Befik und Cigenthum im artge= meinen . . . . . . . . . . . . 315

$3 \mathfrak{w e i t e r} \mathfrak{i} \mathfrak{b} ; \mathfrak{d} \mathfrak{n}$ itt. Ciniges vom (Eigentlyum an betweg= lichen હachen . . . . . . . . . . . 351

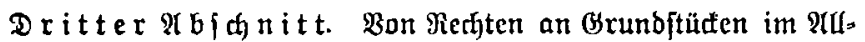
gemcinen . . . . . . . . . 372

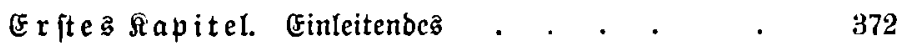

3 reites $\mathfrak{A}$ apitel. Die Brunbbudfeinridtung . . $\quad 376$

Drittes sapitel. Erwerb $2 c$ : von Eigenthum unb

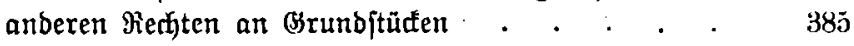

Biertes Siapitel. Shpothet; Brundidyuld; Renten. idjuld . . . . . . . . . . . . . 399

Fünftes siapitel. Fianbrecht an bcroeglifjen હachen. und Redften . 
Fiinfte *tbtheilumg.

Seite

Berf́iedenes a us bem Medteder Gquld= $\mathfrak{v e \mathfrak { h } a ̈ l t n i j 门 e . ~ . ~ . ~ . ~ . ~ . ~ . ~ . ~} 419$

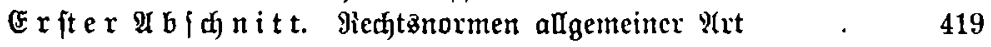

Erite $\mathfrak{B}$ fi a pitel. Willenzerflärungen . . . . $\quad 419$

3reites Sipitel. Bertretung. Bollmadt. Cinrilligung. Benehmigung . . . . . . . 426

Drittes Mapitel. Friften und Termine . . . 430

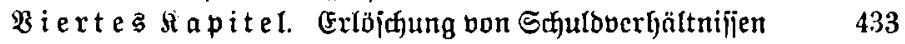

Fünftes $\mathfrak{A}$ apitel. Eicherheitzlleiftung . . . . . 461

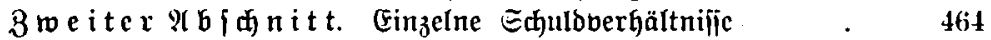

Erftes $\mathfrak{X}$ a pitcl. Einlcitendes . . . . 464

Breites $\mathfrak{H a p i t e l . ~ M i e t h e ~ u n d ~} \mathfrak{B a r h t}$. 469

Irittes $\mathfrak{X}$ apitel. Dienjtocrtrag . . 492

Biertes sapitel. Mertuertrag . . 51;

Fünftes Ha pitel. Mätlerroejen . . . . . . . 5;30

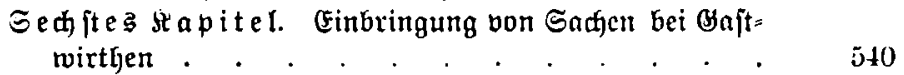

Sicbentes Stapitel. Spiel und Sotterie . $\quad 549$

Átes $\mathfrak{x a p i t e l}$. Jnhaberpapiere . . . . 557

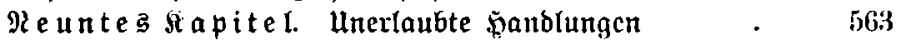

\section{Eedite Whtheilung.}

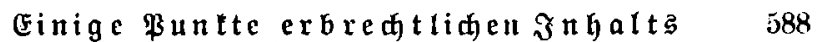

Erites fiapitel. Finleitendes . . . . . 588

3 weites siapitel. Bejebliche crofolge. . . $\quad 590$

Dritte $\mathfrak{A}$ apitel. Benillërte Erbjolge . . . $\quad 594$

Biertes Mapitel. Eidjerung bes Mardalafles . . $\quad$ 598

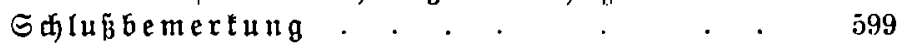

